\title{
Correctness Proofs of Distributed Termination Algorithms
}

\author{
KRZYSZTOF R. APT \\ L.I.T.P., Université Paris 7
}

The problem of correctness of the solutions to the distributed termination problem of Francez [7] is addressed. Correctness criteria are formalized in the customary framework for program correctness. A very simple proof method is proposed and applied to show correctness of a solution to the problem. It allows us to reason about liveness properties of temporal logic (see, e.g., Manna and Pnueli [12]) using a new notion of weak total correctness.

Categories and Subject Descriptors: D.1.3 [Programming Techniques]: Concurrent Programming; F.3.1 [Logics and Meanings of Programs]: Specifying and Verifying and Reasoning about Programs.

General Terms: Algorithms, Theory, Verification

Additional Key Words and Phrases: CSP, deadlock, global invariant, weak total correctness

\section{INTRODUCTION}

This paper deals with the distributed termination problem of Francez [7] which has received a great deal of attention in the literature. Several solutions to this problem, or its variants, have been proposed, their correctness, however, has been rarely discussed. In fact, it is usually not even explicitly stated what properties such a solution should satisfy.

Notable exceptions in this matter are papers of Dijkstra, Feijen, and Van Gasteren [5] and Topor [14] in which solutions to the problem are systematically derived together with their correctness proofs. On the other hand, they are presented in a simplistic abstract setting in which, for example, no distinction can be made between deadlock and termination. Also, as we shall see in the next section, not all desired properties of a solution are addressed there. Systematically derived solutions in the abstract setting of [5] are extremely helpful in understanding corresponding solutions presented in CSP. However, such a presentation should not relieve us from providing rigorous correctness proofs-an issue we address in this paper.

A preliminary version of this paper appeared in Logics and Models of Concurrent Systems, K. R. Apt, Ed., Springer Verlag, NATO ASI Series, vol. F13, 1985.

Author's address: L.I.T.P. Université Paris 7, 2, Place Jussieu, 75251 Paris, France.

Permission to copy without fee all or part of this material is granted provided that the copies are not made or distributed for direct commercial advantage, the ACM copyright notice and the title of the publication and its date appear, and notice is given that copying is by permission of the Association for Computing Machinery. To copy otherwise, or to republish, requires a fee and/or specific permission.

(C) 1986 ACM 0164-0925/86/0700-0388 $\$ 00.75$

ACM Transactions on Programming Languages and Systems, Vol. 8, No. 3, July 1986, Pages 388-405. 
Clearly, it would be preferable to derive the solutions in CSP together with their correctness proofs, perhaps by transforming accordingly the solutions first provided in the abstract setting. Unfortunately, such techniques are not presently available.

This paper is organized as follows. In the next section we define the problem and propose the correctness criteria that the solutions to the problem should satisfy. Then, in Section 3, we formalize these criteria in the usual framework for program correctness, and in Section 4 we propose a very simple proof method which allows us to prove the criteria. In Section 5 we provide a simple solution to the problem, and in the next section give a detailed proof of its correctness. Finally, in section Section 7, we assess the proposed proof method.

Throughout this paper we assume that the reader is familiar with the language of Communicating Sequential Processes (CSP), as defined in Hoare [10], and has some experience in the proofs of correctness of very simple loop-free sequential programs.

\section{DISTRIBUTED TERMINATION PROBLEM}

Suppose that a CSP program

$$
P=\left[P_{1}\|\ldots\| P_{n}\right]
$$

where for every $\mathbf{1} \leq i \leq n, P_{i}:: \operatorname{INIT}_{i}:{ }^{*}\left[S_{i}\right]$ is given. We assume that each $S_{i}$ is of the form $\underset{j \in \Gamma_{i}}{\square} g_{i, j} \rightarrow S_{i, j}$ for an index set $\Gamma_{i}$ and that

(i) each $g_{i, j}$ contains an I/O command addressing $P_{j}$,

(ii) none of the statements $\mathrm{INIT}_{i}, S_{i, j}$ contains an I/O command.

We say then that $P$ is in a normal form. Suppose moreover that we associate with each $P_{i}$ a stability condition, $B_{i}$, a Boolean expression involving variables of $P_{i}$ and possibly some auxillary variables. By a global stability we mean a situation in which each process is at the main loop entry with its stability condition $B_{i}$ true.

We now adopt the following two assumptions:

(a) No communication can take place between a pair of processes while both of their stability conditions hold.

(b) Whenever deadlock takes place the global stability condition is reached.

The distributed termination problem (see Francez [7]) is the problem of transforming $P$ into another program $P^{\prime}$ which eventually properly terminates whenever the global stability condition is reached. Its formulation presupposes that $P^{\prime}$ has a particular format so that each computation of $P^{\prime}$ can be restricted to a computation of $P$ by omitting the instructions involving new variables.

We say that the global stability condition is (not) reached in a computation of $P^{\prime}$ if it is (not) reached in its restriction to a computation of $P$. In turn, the global stability condition is reached (not reached) in a computation of $P$ if it holds (does not hold) in a (any) possible global state of the computation. We consider here partially ordered computations in the sense of [11]. 
We now postulate four properties that a solution $P^{\prime}$ to the distributed termination problem should satisfy (see Apt and Richier [4]):

(1) Whenever $P^{\prime}$ properly terminates then the global stability condition is reached.

(2) There is no deadlock.

(3) If the global stability condition is reached then $P^{\prime}$ eventually properly terminates.

(4) If the global stability condition is not reached then eventually a statement from the original program is executed.

The last property excludes the situations in which the transformed parallel program endlessly executes the added control parts dealing with termination detection.

In the abstract framework of [5] only the first property is proved. The second property is not meaningful, as there deadlock coincides with termination. In turn, satisfaction of the third property is argued informally, and the fourth property is not mentioned.

Solutions to the distributed termination problem are obtained by arranging some additional communications between the processes $P_{i}$. Most of them are programs, $P^{\prime}=\left[P_{1}\|\ldots\| P_{n}\right]$, in a normal form where for every $i, 1 \leq i \leq n$,

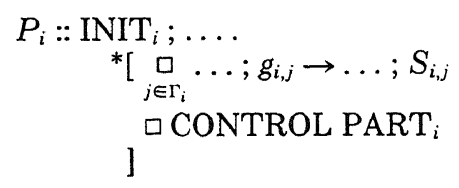

where ... stand for some added Boolean conditions or statements not containing $\mathrm{I} / \mathrm{O}$ commands, and CONTROL PART $\mathrm{P}_{i}$ stands for a part of the loop dealing with additional communications.

For solutions of the above form we now express the four properties which we have introduced, using the customary terminology dealing with program correctness.

\section{FORMALIZATION OF THE CORRECTNESS CRITERIA}

Let $p, q, I$ be assertions from an assertion language and let $S$ be a CSP program. We say that $\{p\} S\{q\}$ holds in the sense of partial correctness if all properly terminating computations of $S$ starting in a state satisfying $p$ terminate in a state satisfying $q$. We say that $\{p\} S\{q\}$ holds in the sense of weak total correctness if it holds in the sense of partial correctness and, moreover, no computation of $S$ starting in a state satisfying $p$ fails or diverges. We say that $S$ is deadlock-free relative to $p$ if in the computations of $S$ starting in a state satisfying $p$ no deadlock can arise. If $p \equiv$ true, then we simply say that $P$ is deadlock-free.

Finally, we say that $\{p\} S\{q\}$ holds in the sense of total correctness if it holds in the sense of weak total correctness and, moreover, $S$ is deadlock-free relative to $p$. Thus, when $\{p\} S\{q\}$ holds in the sense of total correctness, then all computations of $S$ starting in a state satisfying $p$ properly terminate.

Also, for CSP programs in a normal form we introduce the notion of a global invariant $I$. We say that $I$ is a global invariant of $P$ relative to $p$ if in all ACM Transactions on Programming Languages and Systems, Vol. 8, No. 3, July 1986. 
computations of $P$, starting in a state satisfying $p, I$ holds whenever each process $P_{i}$ is at the main loop entry. If $p \equiv$ true, then we simply say that $I$ is a global invariant of $P$.

Now property 1 simply means that

$$
\{\text { true }\} P^{\prime}\left\{\bigwedge_{i=1}^{n} B_{i}\right\}
$$

holds in the sense of partial correctness.

Property 2 means that $P^{\prime}$ is deadlock-free.

Property 3 cannot be expressed by referring directly to the program $P^{\prime}$. Even though it refers to the termination of $P^{\prime}$, it is not equivalent to its (weak) total correctness because the starting point-the global stability situation-is not the initial one. It is a control point that can be reached in the course of a computation.

However, we can still express property 3 by referring to the weak total correctness of a program derived from $P^{\prime}$. Consider the following program:

$$
\begin{aligned}
& \text { CONTROL PART }= \\
& {\left[P_{1}::{ }^{*}\left[\mathrm{CONTROL} \mathrm{PART}_{1}\right]\|\ldots\| P_{n}::{ }^{*}\left[\mathrm{CONTROL} \mathrm{PART}_{n}\right]\right] \text {. }}
\end{aligned}
$$

We now claim that to establish property 3 it is sufficient to prove for an appropriately chosen global invariant $I$ of $P^{\prime}$ that

$$
\left\{I \wedge \bigwedge_{i=1}^{n} B_{i}\right\} \text { CONTROL PART }\{\text { true }\}
$$

in the sense of total correctness.

Indeed, suppose that in a computation of $P^{\prime}$ the global stability condition is reached. Then, $I \wedge \wedge_{i=1}^{n} B_{i}$ holds where $I$ is a global invariant of $P^{\prime}$. By assumption (a), concerning the original program $P$, no statement from $P$ can be executed any more. Thus the part of $P^{\prime}$ that remains to be executed is equivalent to the program CONTROL PART. Now, because of (2), property 3 holds.

Now consider property 4 . As before, we express it by referring to the program CONTROL PART. Assuming that property 2 holds, property 4 clearly holds if

$$
\left\{I \wedge \neg \bigwedge_{i=1}^{n} B_{i}\right\} \text { CONTROL PART }\{\text { true }\}
$$

holds in the sense of weak total correctness. Indeed, (3) guarantees that if the global stability condition is not currently holding, then every segment of the computation that performs actions from the CONTROL PART only must either terminate or deadlock. In the absence of deadlock this is equivalent to property 4.

Assuming that property 2 is already established, to show property 3 it is sufficient to prove (2) in the sense of weak total correctness. Now (2) and (3) can be combined into the formula:

$$
\text { \{I\} CONTROL PART }\{\text { true }\}
$$

in the sense of weak total correctness. 


\section{PROOF METHOD}

We now present a simple proof method which allows us to handle the properties discussed in the previous section. The proof method uses to great advantage the coarse granularity that can be imposed on CSP programs in defining the atomic transitions due to the disjointness of processes. It can only be applied to CSP programs being in a normal form. So assume that $P=\left[P_{1}\|\ldots\| P_{n}\right]$ is such a program.

Given a guard $g_{i, j}$, we denote by $b_{i, j}$ the conjunction of its Boolean parts. We say that guards $g_{i, j}$ and $g_{j, i}$ match if one contains an input command and the other an output command whose expressions are of the same type. The notation implies that these I/O commands address each other (i.e., they are within the texts of $P_{i}$ and $P_{j}$, respectively, and address $P_{j}$ and $P_{i}$, respectively).

Given two matching guards $g_{i, j}$ and $g_{j, i}$, we denote by $\operatorname{Eff}\left(g_{i, j}, g_{j, i}\right)$ the effect of the communication between their I/O commands. It is the assignment whose left-hand side is the input variable and the right-hand side is the output expression. Finally, let

$$
\text { TERMINATED }=\bigwedge_{\substack{1 \leq i \leq n \\ j \in \Gamma_{i}}}^{\wedge} \neg b_{i, j}
$$

Observe that TERMINATED holds upon termination of $P$.

Now consider partial correctness. We propose the following proof rule:

Rule 1. Partial Correctness

$\{p\} \operatorname{INIT}_{1} ; \ldots ; \operatorname{INIT}_{n}\{I\}$

$\left\{I \wedge b_{i, j} \wedge b_{j, i}\right\} \operatorname{Eff}\left(g_{i, j}, g_{j, i}\right) ; S_{i, j} ; S_{j, i}\{I\}$

for all $i, j$ s.t. $i \in \Gamma_{j}, j \in \Gamma_{i}$, and $g_{i, j}, g_{j, i}$ match

$\{p\} P\{I \wedge$ TERMINATED $\}$

This rule has to be used in conjunction with the usual proof system for partial correctness of nondeterministic programs (see, e.g., Apt [1]) in order to be able to establish its premises. If the premises of this rule hold, then we can also deduce that $I$ is a global invariant of $P$ relative to $p$. Informally, it can phrased as follows. If $I$ is established upon execution of all the INIT $_{i}$ sections and is preserved by a joint execution of each pair of branches of the main loops with matching guards, then $I$ holds upon exit. Thus, $I$ holds upon exit for such special "synchronized" computations. Intuitively this rule is sound because due to the disjointness of processes each terminating computation of $P$ can be appropriately rearranged so that it becomes a "synchronized" computation.

Consider now weak total correctness. We adopt the following proof rule:

Rule 2. Weak Total Correctness

$\{p\} \operatorname{INIT}_{1} ; \ldots ; \operatorname{INIT}_{n}\{I \wedge t \geq 0\}$

$\left\{I \wedge b_{i, j} \wedge b_{j, i} \wedge z=t \wedge t \geq 0\right\} \operatorname{Eff}\left(g_{i, j}, g_{j, i}\right) ; S_{i, j} ; S_{j, i}\{I \wedge 0 \leq t<z\}$ for all $i, j$ s.t. $i \in \Gamma_{j}, j \in \Gamma_{i}$, and $g_{i, j}, g_{j, i}$ match

$\{p\} P\{I \wedge$ TERMINATED $\}$

where $z$ does not appear in $P$ or $t$ and $t$ is an integer-valued expression.

ACM Transactions on Programming Languages and Systems, Vol. 8, No. 3, July 1986. 
This rule has to be used in conjunction with the standard proof system for total correctness of nondeterministic programs (see, e.g., Apt [1]) in order to establish its premises. It is a usual modification of the rule concerning partial correctness which guarantees lack of divergence.

Finally, consider deadlock freedom. Let

$$
\mathrm{BLOCKED}=\wedge\left\{\neg b_{i, j} \vee \neg b_{j, i}: 1 \leq i, j \leq n, i \in \Gamma_{j}, j \in \Gamma_{i}, g_{i, j} \text { and } g_{j, i} \text { match }\right\}
$$

Observe that in a given state of $P$ the formula BLOCKED holds if and only if no communication between the processes is possible. We now propose the following proof rule:

Rule 3. Deadlock Freedom

$I$ is a global invariant of $P$ relative to $p$,

$I \wedge$ BLOCKED $\rightarrow$ TERMINATED.

$P$ is deadlock-free relative to $p$.

The above rules are used in conjunction with a rule of auxiliary variables.

Let $A$ be a set of variables of a program $S$. $A$ is called a set of auxiliary variables of $S$ if:

(i) All variables from $A$ appear in $S$ only in assignments.

(ii) No variable of $S$ which is not in $A$ depends on the variables from $A$. In other words, there does not exist an assignment $x:=t$ in $S$ such that $x \notin A$ and $t$ contains a variable from $A$.

Thus, for example, $\{z\}$ and $\{y, z\}$ are the only nonempty sets of auxiliary variables of the program

$$
\left[P_{1}:: z:=y ; P_{2} ! \times \| P_{2}:: P_{1} ? u ; u:=u+1\right] \text {. }
$$

We now adopt the following proof rule first introduced in Owicki and Gries [13].

\section{Rule 4. Auxiliary Variables}

Let $A$ be a set of auxiliary variables of a program $S$. Let $S^{\prime}$ be obtained from $S$ by deleting all assignments to the variables in $A$. Then,

$$
\frac{\{p\} S\{q\}}{\{p\} S^{\prime}\{q\}}
$$

provided $q$ has no free variable from $A$.

Also, if $S$ is deadlock-free relative to $p$, then so is $S^{\prime}$.

We use this rule both in the proofs of partial and of (weak) total correctness. Also, we freely use the well-known consequence rule which allows us to strengthen the preconditions and weaken the postconditions of a correctness statement $\{p\} S\{q\}$.

\section{A SOLUTION}

We now present a simple solution to the distributed termination problem. It is a combination of the solutions proposed by Francez, Rodeh, and Sintzoff [8] and (in an abstract setting) Dijkstra, Feijen, and Van Gasteren [5]. 
We assume that the graph consisting of all communication channels within $P$ contains a Hamiltonian cycle (i.e., a communication path visiting each process exactly once). In the resulting ring the neighbors of $P_{i}$ are $P_{i-1}$ and $P_{i+1}$, where counting is done within $\{1, \ldots, n\}$ cyclically.

We first present a solution in which the global stability condition is detected by one process, say $P_{1}$. It has the following form, where the newly introduced variables $s_{i}$, send $_{i}$, and $\operatorname{moved}_{i}$ do not appear in the original program $P$ :

For $i=1$

$P_{i}:: \mathrm{INIT}_{i} ;$ send $_{i}:=$ true ;

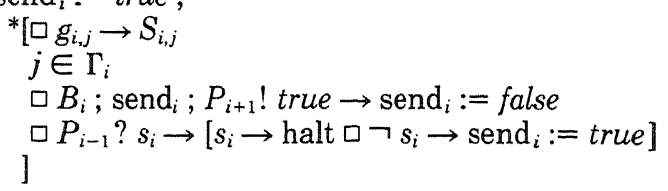

and, for $i \neq 1$,

$P_{i}::$ send $\mathrm{INIT}_{i} ;:=$ false $; \operatorname{moved}_{i}:=$ false $:$

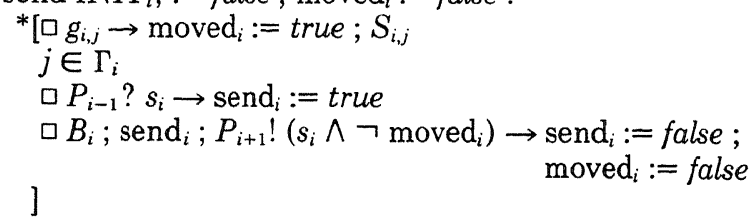

In this program we use the halt instruction with an obvious meaning. Informally, $P_{1}$ decides to send a probe true to its right-hand side neighbor when its stability condition $B_{1}$ holds. A probe can be transmitted by a process $P_{i}$ further to its right-hand neighbor when in turn its stability condition holds. Each process writes into the probe, its current status being reflected by the variable moved; moved turns to true when a communication from the original program takes place and turns to false when the probe is sent to the right-hand side neighbor.

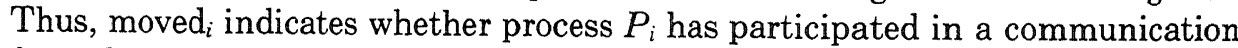
from the original program since the last time it had passed the probe. $P_{1}$ decides to stop its execution when a probe has made a full cycle remaining true. This will happen if all the moved variables are false at the moment of receiving the probe from the left-hand side neighbor.

We now modify this program by arranging that $P_{1}$ send a final termination wave through the ring once it detects the global stability condition. To this purpose, we introduce in all $P_{i}$ s two new Boolean variables, detected ${ }_{i}$ and done $e_{i}$. The program has the following form:

For $i=1$

$P_{i}::$ done $_{i}$ send $_{i}:=$ true $;$ done $_{i}:=$ false $;$ detected $_{i}:=$ false

${ }^{*}\left[\square \operatorname{INIT}_{i j}\right.$ done $_{i}, g_{i, j} \rightarrow S_{i, j}$

$j \in \Gamma_{p}$

$\square \neg$ done $_{i} ; B_{i} ;$ send $_{i} ; P_{i+1}$ ! true $\rightarrow$ send $_{i}:=$ false

$\square \neg$ done $_{i} ; P_{i-1}$ ? $s_{i} \rightarrow$

$\left[s_{i} \rightarrow \operatorname{detected}_{i}:=\right.$ true $\square \neg s_{i} \rightarrow \operatorname{send}_{i}:=$ true $]$

$\square$ detected $_{i} ; P_{i+1}$ ! end $\rightarrow$ detected $_{i}:=$ false ]

$\neg$ done $_{i} ; P_{i-1}$ ? end $\rightarrow$ done $_{i}:=$ true

ACM Transactions on Programming Languages and Systems, Vol. 8, No. 3, July 1986. 
and for $i \neq 1$

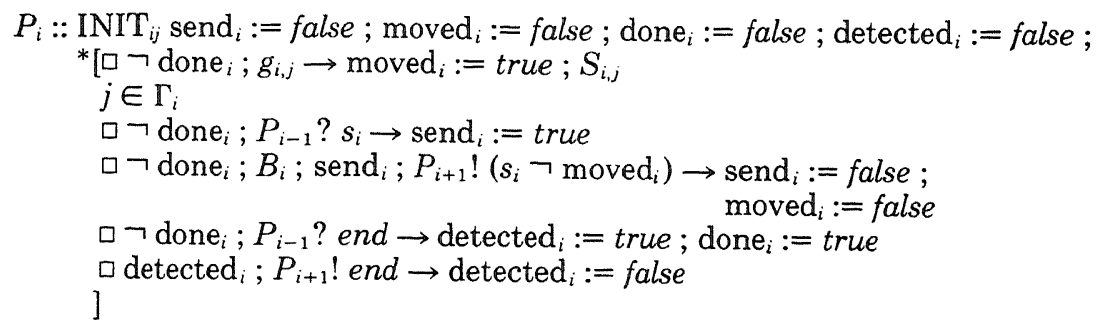

We assume that end is a signal of a new type not used in the original program. (Actually, to avoid confusion in the transmission of the probe, we also have to assume that in the original program no messages are of type Boolean. If this is not the case, then we can always replace the probe by a Boolean-valued message of a new type.)

\section{CORRECTNESS PROOF}

We now prove correctness of the solution given in the previous section using the proof method introduced in Section 4 . We do this by proving properties $1-4$ of Section 2 as formalized in Section 3.

Proof of Property 1. We first modify the program given in the previous section by introducing in process $P_{1}$ auxiliary variables received and forward $_{1}$. The variable received ${ }_{1}$ is introduced in order to distinguish the situation when $s_{1}$ is initially true from the one when $s_{1}$ turns true after the communication with $P_{n}$. The variable forward $d_{1}$ is used to remember whether or not $P_{1}$ sent the end signal to $P_{2}$. Note that this fact cannot be expressed by referring to the variable detected $_{1}$. This augmented version of $P_{1}$ has the following form:

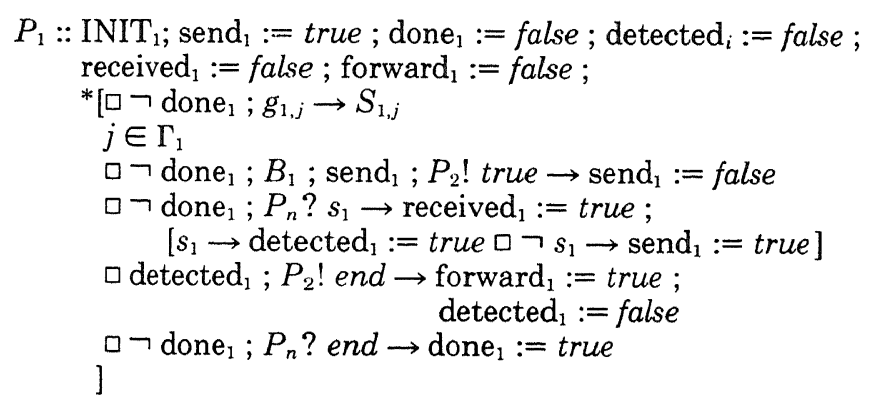

Other processes remain unchanged. Call this modified program $R$. In order to establish property 1 , it is sufficient, by rule 4 , to find a global invariant of $R$ which upon its termination implies $\wedge_{i=1}^{n} B_{i}$.

We do this by establishing a sequence of successively stronger global invariants whose final element is the desired $I$. We call a program $\operatorname{Eff}\left(g_{i, j}^{\prime}, g_{j, i}^{\prime}\right) ; S_{i, j}^{\prime} ; \mathbf{S}_{j, i}^{\prime}$, corresponding to a joint execution of two branches of the main loops with matching guards in a program being in a normal form, a transition. For a convenient expression of loop invariants, we identify the Boolean values false, true with 0 and 1 , respectively. To avoid excessive use of brackets we assume 
that " $\rightarrow$ " binds weaker than other connectives. Let

$$
I_{1} \equiv \sum_{i=1}^{n} \operatorname{send}_{i} \leq 1 .
$$

Then $I_{1}$ is clearly a global invariant of $R$ : it is established by the initial assignments and is preserved by every transition, as the setting of a send variable to true is always accompanied by changing another send variable from true to false. Now consider

$$
\begin{aligned}
I_{2} \equiv & \forall i>1\left[s_{i} \wedge \operatorname{send}_{i} \rightarrow \forall j\left(1 \leq j<i \rightarrow B_{j}\right) \vee \exists j \geq i \operatorname{moved}_{j}\right] \\
& \wedge\left[s_{1} \wedge \text { received }_{1} \rightarrow \forall j\left(1 \leq j \leq n \rightarrow B_{j}\right)\right] .
\end{aligned}
$$

We now claim that $I_{1} \wedge I_{2}$ is a global invariant of $R$. First note that $I_{2}$ is established by the initial assignments in a trivial way.

Next, consider a transition corresponding to a communication from the original program $P$. Assume that initially $I_{1} \wedge I_{2}$ and that the Boolean conditions of the guards hold.

Now consider the first conjunct of $I_{2}$. If, initially, for no $i>1$ does $s_{i} \wedge$ send $_{i}$ hold, then this conjunct is preserved since the transition does not alter $s_{i}$ or $\operatorname{send}_{i}$. Suppose now that initially for some $i>1, s_{i} \wedge$ send $_{i}$ holds. If initially also $\exists j \geq i$ moved $_{i}$ holds, then this conjunct is preserved. If initially $\forall j\left(1 \leq j<i \rightarrow B_{j}\right)$ holds, then, by assumption (a) in Section 2 , at least one of the processes involved in the transition has an index $\geq i$. The transition sets its moved variable to true which establishes $\exists j \geq i$ moved $_{j}$.

The second conjunct of $I_{2}$ is obviously preserved-if initially $s_{1} \wedge$ received $_{1}$ does not hold, then it does not hold at the end of the transition either. If initially $s_{1} \wedge$ received $_{1}$ holds, then also $\forall j\left(1 \leq j \leq n \rightarrow B_{j}\right)$ initially holds, and again, by assumption (a) of Section 2, the discussed transition cannot take place.

Now consider a transition corresponding to a sending of the probe from $P_{i}$ to $P_{i+1}(1 \leq i \leq n)$. Suppose that at the end of the transition $s_{k} \wedge$ send $_{k}$ for some $k$ $(1<k \leq n)$ holds. Due to the global invariant $I_{1}$ and the form of the transition, we can conclude that $k=i+1$. Thus, in the initial state, $B_{i} \wedge s_{i} \wedge \neg \operatorname{moved}_{i} \wedge$ $\operatorname{send}_{i}$ holds. Now, due to $I_{2}$, initially

holds. Thus, initially

$$
\forall j\left(1 \leq j<i \rightarrow B_{j}\right) \vee \exists j \geq i \text { moved }_{j}
$$

$$
\forall j\left(1 \leq j<i+1 \rightarrow B_{j}\right) \vee \exists j \geq i+1 \text { moved }_{j}
$$

holds. This formula is not affected by the execution of the transition. Thus, at the end of the transition, the first conjunct of $I_{2}$ holds.

Now suppose that at the end of the transition $s_{1} \wedge$ received holds. If initially $s_{1} \wedge$ received $_{1}$ holds, then also $\forall j\left(1 \leq j \leq n \rightarrow B_{j}\right)$ initially holds. Now suppose that initially $s_{1} \wedge$ received $_{1}$ does not hold. Then the transition consists of sending the probe from $P_{n}$ to $P_{1}$. Therefore, initially $B_{n} \wedge s_{n} \wedge \neg \operatorname{moved}_{n} \wedge$ send $_{n}$ holds, and because of $I_{2}, \forall j\left(1 \leq j \leq n \rightarrow B_{j}\right)$ initially holds as well. But this formula is preserved by the execution of the transition. So at the end of the transition the

ACM Transactions on Programming Languages and Systems, Vol. 8, No. 3, July 1986 
The other transitions do not affect $I_{2}$. So $I_{1} \wedge I_{2}$ is indeed a global invariant of $R$. We do not have as yet that, upon termination of $R, I_{1} \wedge I_{2}$ implies $\wedge_{i=1}^{n} B_{i}$. But it is now sufficient to show that upon termination of $R$ the conjunction $s_{1} \wedge$ received $_{1}$ holds.

Now consider

$$
I_{3} \equiv \text { detected }_{1} \rightarrow s_{1} \wedge \text { received }_{1} .
$$

It is straightforward to see that $I_{3}$ is a global invariant of $R$. Next, let

$$
I_{4} \equiv \text { forward }_{1} \rightarrow s_{1} \wedge \text { received }_{1} .
$$

Then $I_{3} \wedge I_{4}$ is a global invariant of $R$. Indeed, when forward ${ }_{1}$ becomes true, initially detected ${ }_{1}$ holds, so by virtue of $I_{3}, s_{1} \wedge$ received $_{1}$ initially holds. But $s_{1} \wedge$ received $_{1}$ is not affected by the execution of the transition in question.

Now we show that, upon termination of $R$, forward ${ }_{1}$ holds. To this end, consider

$$
I_{5} \equiv \text { done }_{2} \rightarrow \text { forward }_{1} .
$$

Clearly, $I_{5}$ is a global invariant: done $_{2}$ and forward ${ }_{1}$ become true in the same transition. Now let

$$
I \equiv \bigwedge_{i=1}^{5} I_{i}
$$

Then $I$ is the desired global invariant: upon termination of $R$, done $e_{2}$ holds, and done $_{2} \wedge I$ implies $\wedge_{i=1}^{n} B_{i}$.

Proof of Property 2. We now also modify processes $P_{i}$ for $i \neq 1$, by introducing in each of them the auxiliary variable forward ${ }_{i}$ for the same reasons as in $P_{1}$.

The augmented versions of $P_{i}(i \neq 1)$ have the following form:

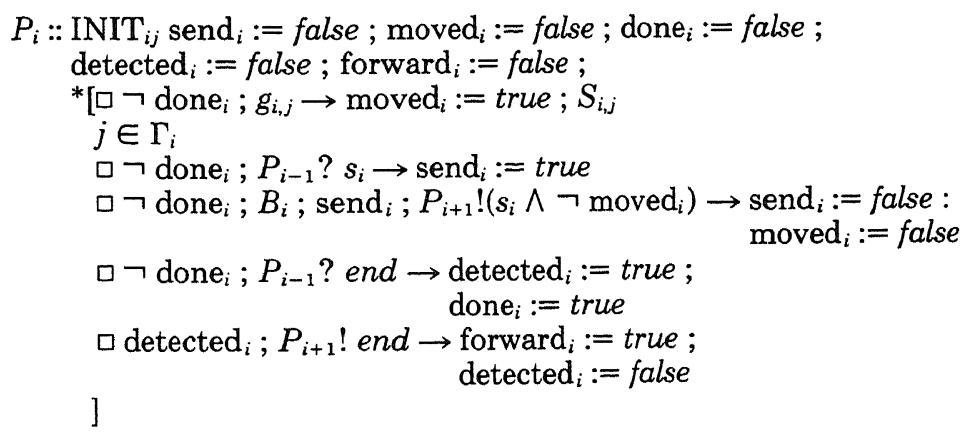

Call this augmented version of the program $S$. We now prove that $S$ is deadlockfree. In the subsequent proofs it will be more convenient to consider the second premise of rule 3 in the equivalent form:

$$
I \wedge \neg \text { TERMINATED } \rightarrow \neg \text { BLOCKED. }
$$

Let, for $i=1, \ldots, n$,

TERMINATED $_{i} \equiv$ done $_{i} \wedge \neg$ detected $_{i}$.

ACM Transactions on Programming Languages and Systems, Vol. 8, No. 3, July 1986. 
Note that, if in a deadlock situation of $S$, TERMINATED ${ }_{i}$ holds, then $P_{i}$ has terminated. The following natural decomposition of $\neg$ TERMINATED allows us to carry out a case analysis.

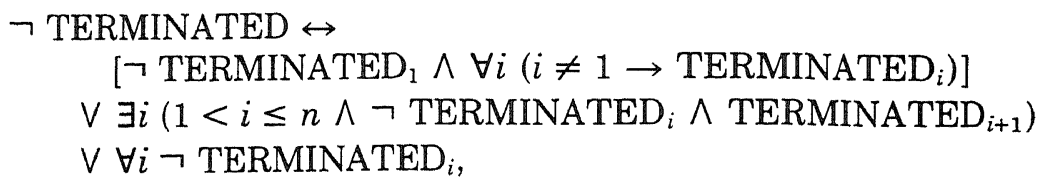

where due to our convention of cyclic counting within $\{1, \ldots, n\}, n+1$ is identified with 1 .

Case 1. It corresponds to a deadlock situation in which $P_{1}$ did not terminate and all $P_{i}$ for $i \neq 1$ have terminated. Let

$$
\begin{aligned}
& I_{6} \equiv \neg \text { detected }_{n} \wedge \text { done }_{n} \rightarrow \text { forward }_{n}, \\
& I_{7} \equiv \text { forward }_{n} \rightarrow \text { done }_{1} .
\end{aligned}
$$

It is straightforward to see that $I_{6}$ and $I_{7}$ are global invariants of $S$. Now let

$$
\begin{aligned}
I_{8} & \equiv \text { done }_{2} \rightarrow \text { forward }_{1}, \\
I_{9} & \equiv \text { detected }_{1} \rightarrow \sum_{i=1}^{n} \operatorname{send}_{i}=0, \\
I_{10} & \equiv \text { forward }_{1} \rightarrow \sum_{i=1}^{n} \operatorname{send}_{i}=0, \\
I_{11} & \equiv \text { forward }_{1} \rightarrow \neg \text { detected }_{1} .
\end{aligned}
$$

Then $I_{8}, I_{9}, I_{9} \wedge I_{10}, I_{9} \wedge I_{10} \wedge I_{11}$ are all global invariants of $S$. To see this, consider by way of example $I_{9} \wedge I_{10} \wedge I_{11}$ under the assumption that $I_{9} \wedge I_{10}$ is already shown to be a global invariant. It is obviously established by the initial assignments of $S$. In view of the invariance of $I_{9} \wedge I_{10}$, the only transition which can falsify $I_{9} \wedge I_{10} \wedge I_{11}$ is the one involving reception of the probe by $P_{1}$. But then intially send $d_{n}$ holds, so by $I_{10}$ initially $\neg$ forward ${ }_{1}$ holds. The transition does not change the value of forward $d_{1}$. So forward $d_{1}$ remains false and $I_{11}$ holds at the end of the transition. Now let

$$
J=\bigwedge_{i=6}^{11} I_{i}
$$

$J$ is a global invariant of $S$. Observe now that

$$
J \wedge \text { TERMINATED }_{n} \rightarrow \text { done }_{1}
$$

due to $I_{6}$ and $I_{7}$, and

$$
J \wedge \text { TERMINATED }_{2} \rightarrow \neg \text { detected }_{1}
$$

due to $I_{8}$ and $I_{11}$. Thus,

$$
J \wedge \text { TERMINATED }_{2} \wedge \text { TERMINATED }_{n} \rightarrow \text { TERMINATED }_{1} .
$$

That is,

$$
J \wedge\left[\neg \text { TERMINATED }_{1} \wedge \forall i\left(i \neq 1 \rightarrow \text { TERMINATED }_{i}\right)\right]
$$

is unsatisfiable.

ACM Transactions on Programming Languages and Systems, Vol. 8, No. 3, July 1986. 
Case 2. This corresponds to a deadlock situation in which for some $i$, $1<i \leq n, P_{i}$ did not terminate, whereas $P_{i+1}$ has terminated. Let some $i$, $1<i \leq n$, be given. Let

$$
\begin{aligned}
& I_{12} \equiv \text { done }_{i+1} \rightarrow \text { forward }_{i}, \\
& I_{13} \equiv \operatorname{detected}_{i} \rightarrow \text { done }_{i}, \\
& I_{14} \equiv \text { forward }_{i} \rightarrow \text { done }_{i} \wedge \neg \operatorname{detected}_{i} .
\end{aligned}
$$

It is straightforward to see that $I_{12}, I_{13}$, and $I_{13} \wedge I_{14}$ are global invariants. Let

$$
K \equiv I_{12} \wedge I_{13} \wedge I_{14} \text {. }
$$

Then $K$ is a global invariant and

$$
K \wedge \text { TERMINATED }_{i+1} \rightarrow \text { TERMINATED }_{i}
$$

results from $I_{12}$ and $I_{14}$. Thus

$$
K \wedge \neg \text { TERMINATED }_{i} \wedge \text { TERMINATED }_{i+1}
$$

is unsatisfiable.

In fact, we show that neither case 1 nor case 2 can arise.

Case 3. This corresponds to a deadlock situation in which none of the processes have terminated. Let

$$
I_{15} \equiv \text { done }_{1} \rightarrow \text { forward }_{n} .
$$

$I_{15}$ is a global invariant. Also, $I_{12}$ for all $i$ s.t. $1<i<n$ and $I_{13} \wedge I_{14}$ for all $i$ s.t. $1<i \leq n$ are global invariants. Let

$$
L \equiv I_{15} \wedge \bigwedge_{i=2}^{n-1} I_{12} \wedge \bigwedge_{i=2}^{n}\left(I_{13} \wedge I_{14}\right)
$$

Then $L$ is a global invariant and

$$
L \wedge \exists i\left(i \neq 2 \wedge \text { done }_{i}\right) \rightarrow \exists i \text { TERMINATED }{ }_{i},
$$

due to $I_{15}, \wedge_{i=2}^{n-1} I_{12}$ and $\bigwedge_{i=2}^{n} I_{14}$. Thus,

$$
L \wedge \forall i \neg \text { TERMINATED } \rightarrow \forall i\left(i \neq 2 \rightarrow \neg \text { done }_{i}\right) .
$$

Hence,

$$
L \wedge \forall i \neg \text { TERMINATED }_{i} \wedge \text { done }_{2}
$$$$
\rightarrow \text { detected }_{2} \wedge \neg \text { done }_{3} \rightarrow
$$

$\neg$ BLOCKED, since $P_{2}$ and $P_{3}$ can communicate.

It remains to consider the case when $\neg$ done $_{2}$ holds. Let

$$
\begin{aligned}
I_{16} & \equiv \sum_{i=1}^{n} \operatorname{send}_{i}=0 \rightarrow s_{1} \wedge \text { received }_{1}, \\
I_{17} & \equiv s_{1} \wedge \operatorname{received}_{1} \wedge \neg \operatorname{detected}_{1} \rightarrow \text { forward }_{1}, \\
I_{18} & \equiv \text { forward }_{1} \rightarrow \text { done }_{2} .
\end{aligned}
$$

Then $I_{16}, I_{17}$, and $I_{18}$ are global invariants. 
Let $\operatorname{BLOCKED~}(P)$ stand for the formula BLOCKED (defined in Section 4) constructed for the original program $P$ from Section 2. Assumption (b) of Section 2 states that whenever deadlock is reached, the global stability condition holds, and simply means that

$$
\phi \equiv \operatorname{BLOCKED}(P) \rightarrow \bigwedge_{i=1}^{n} B_{i}
$$

is a global invariant of $P$. But by the form of $S, \phi$ is also a global invariant of $S$, as the added transitions do not alter the variables of $P$. Thus

$$
M \equiv L \wedge I_{16} \wedge I_{17} \wedge I_{18} \wedge \phi
$$

is a global invariant of $S$.

We now have

$M \wedge \forall i \neg$ TERMINATED $_{i} \wedge \neg$ done $_{2} \wedge$ BLOCKED $\rightarrow$ (by (5))

$M \wedge \forall i \neg$ done $_{i} \wedge$ BLOCKED $\rightarrow$ (by the form of $S$ )

$M \wedge \forall i \neg$ done $_{i} \wedge \operatorname{BLOCKED} \wedge \operatorname{BLOCKED}(P) \rightarrow($ since $\phi$ is a part of $M)$

$M \wedge \forall i \neg$ done $_{i} \wedge$ BLOCKED $\wedge \wedge_{i=1}^{n} B_{i} \rightarrow$ (by the form of $S$ )

$M \wedge \forall i \neg$ done $_{i} \wedge \sum_{i=1}^{n}$ send $_{i}=0 \wedge \neg$ detected $_{1} \rightarrow\left(\right.$ since $I_{16}, I_{17}$, and

$I_{18}$ are parts of $M$ )

$\forall i \neg$ done $_{i} \wedge$ done $_{2}$

which is a contradiction. This simply means that

$$
M \wedge \forall i \neg \text { TERMINATED }_{i} \wedge \neg \text { done }_{2} \rightarrow \neg \text { BLOCKED }
$$

which concludes the proof of case 3 .

By rule 3, $S$ is now deadlock-free where $J \wedge K \wedge M$ is the desired global invariant. By rule $4, P^{\prime}$ is deadlock-free.

Proof of Properties 3 and 4. As shown in Section 3, it suffices to find a global invariant $I$ such that $\{I\}$ CONTROL PART $\{$ true $\}$ holds in the sense of weak total correctness.

We first modify the program CONTROL PART by introducing in process $P_{1}$ an auxiliary variable count $t_{1}$ which is used to count the number of times process $P_{1}$ has received the probe. Other processes remain unchanged. Thus, the processes have the following form:

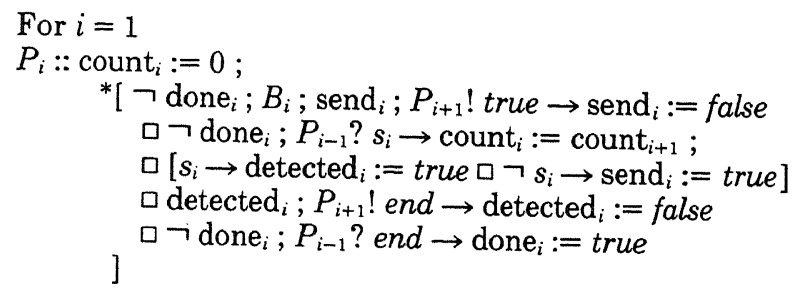

ACM Transactions on Programming Languages and Systems, Vol. 8, No. 3, July 1986. 
and for $i \neq 1$

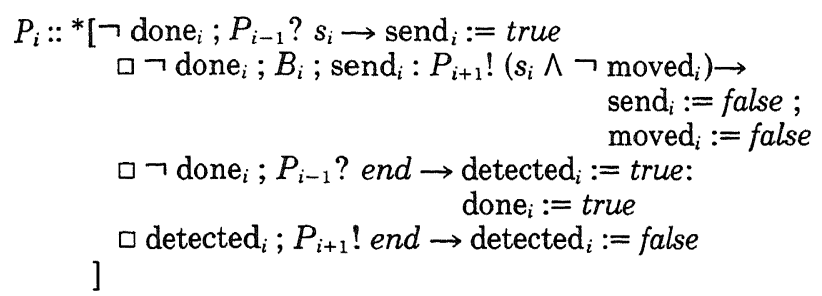

We call this program $T$. For the global invariant $I$ of $P^{\prime}$, we choose the formula $I_{1}$ (i.e., $\sum_{i=1}^{n}$ send $_{i} \leq 1$ ). We now establish a sequence of successively stronger global invariants of $T$ (all relative to $I_{1}$ ). $I_{1}$ itself is clearly a global invariant of $T$. Let

$$
I_{19} \equiv \forall i>1\left[\text { count }_{1}=1 \wedge \operatorname{send}_{i} \rightarrow \forall j\left(1<j<i \rightarrow \neg \operatorname{moved}_{j}\right]\right.
$$

$I_{1} \wedge I_{19}$ is clearly a global invariant of $T$. First, it is obviously established by the

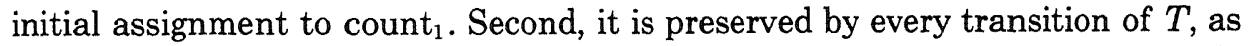
no moved $_{j}$ variable is ever set to true, and whenever a send $d_{i}$ variable for $i>2$ is set to true, then moved $\mathrm{d}_{i-1}$ is set of false. Also, when count $t_{1}$ becomes 1 , then send becomes true and, by invariance of $I_{1}$, no send $d_{i}$ for $i>1$ can be true. Let

$$
I_{20} \equiv \text { count }_{1}=2 \rightarrow \forall j\left(1<j \leq n \rightarrow \neg \operatorname{moved}_{j}\right) .
$$

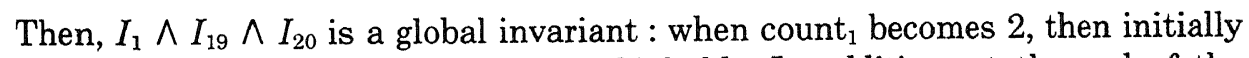
due to $I_{19}, \forall j\left(1<j<i \rightarrow \neg\right.$ moved $\left._{j}\right)$ holds. In addition, at the end of the

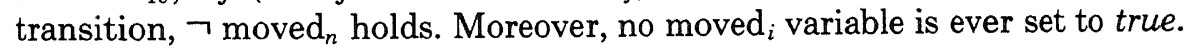

Let

$$
I_{21} \equiv \forall i>1\left(\text { count }_{1}=2 \wedge \operatorname{send}_{i} \rightarrow s_{i}\right) \text {. }
$$

Now consider $I_{1} \wedge \wedge_{j=19}^{21} I_{j}$ and suppose that, by an execution of a transition, send $_{i}$ is set to true when count $t_{1}=2$. If $i=2$, then $s_{2}$ holds, as $s_{2}$ is always set to true. So assume that $i>2$. Then, initially, by $I_{20}$ and $I_{21}, s_{i-1} \wedge \neg \operatorname{moved}_{i-1}$ holds. At the end of the transition, $s_{i}=s_{i-1} \wedge \neg \operatorname{moved}_{i-1}$, so $s_{i}$ holds as desired.

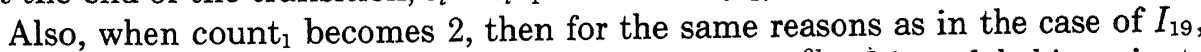
no send $d_{i}$ for $i>1$ can be true. This shows that $I_{1} \wedge \wedge_{j=19}^{21} \dot{I}_{j}$ is a global invariant.

Now let

$$
I_{22} \equiv \operatorname{count}_{1}=3 \rightarrow \sum_{i=1}^{n} \operatorname{send}_{i}=0 \text {. }
$$

Then, $I_{1} \wedge \wedge_{j=19}^{22} I_{j}$ is a global invariant. Indeed, when at the end of a transition

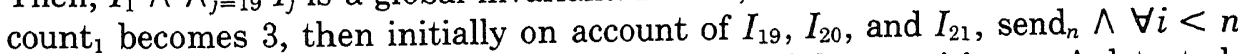
$\neg \operatorname{send}_{i} \wedge s_{n} \wedge \neg \operatorname{moved}_{n}$ holds. Thus, at the end of the transition, $s_{1} \wedge$ detected $_{1}$ $\wedge \forall i \neg$ send $_{i}$ holds.

Also, $\sum_{i=1}^{n}$ send $_{i}=0$ is preserved by every transition. Finally, let

$$
I_{23} \equiv \text { count }_{1} \leq 3 \text {. }
$$

ACM Transactions on Programming Languages and Systems, Vol. 8, No. 3, July 1986. 
Then

$$
N \equiv I_{1} \wedge \bigwedge_{j=19}^{23} I_{j}
$$

is a global invariant of $T$.

Indeed, when at the beginning of a transition, count $t_{1}$ is 3 , then on account of

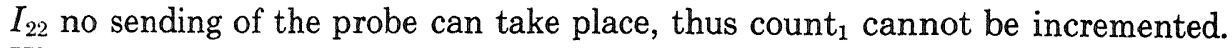
We thus showed that count $t_{1}$ is bounded.

We can now prove formula (4) of Section 3. Indeed, consider the premises of rule 2 for the program $T$. Choose $I_{1}$ for $p, I_{1}$, the global invariant $N$ of $T$, for $I$, and the expression

$$
\left.5 n+3-\left[(n+1) \operatorname{count}_{1}+\sum_{i=1}^{n} \text { done }_{i}+\text { holds (send }\right)\right]
$$

for $t$, where holds(send) is the smallest $j$ for which send ${ }_{j}$ holds if it exists, and 0 otherwise.

We have already shown that $N$ is a global invariant. It is thus sufficient to show that $t$ is always nonnegative and is decremented by each transition. But for all $b_{i, j}$ and $b_{j, i}$ mentioned in the premises of rule 2 ,

$$
N \wedge b_{i, j} \wedge b_{j, i} \rightarrow t>0,
$$

so $t$ is initially positive. Clearly, $t$ is decremented by every transition and

$$
N \rightarrow t \geq 0,
$$

so $t$ remains nonnegative after every transition. Thus by rule 2 ,

$$
\{p\} T\{\text { true }\}
$$

holds in the sense of weak total correctness, so by rule 4, formula (4) from Section 3 holds.

This concludes the correctness proof.

\section{ASSESSMENT OF THE PROOF METHOD}

The proof method proposed in Section 4 is so strikingly simple to state that it is perhaps useful to assess it and to compare it critically with other approaches to proving the correctness of CSP programs. First of all, we should explain why the introduced rules are sound.

Rules 1 and 2 are sound because the CSP programs considered in Section 4 are equivalent to a certain type of nondeterministic program. Namely, consider a CSP program $P$ of the form introduced in Section 2. Let

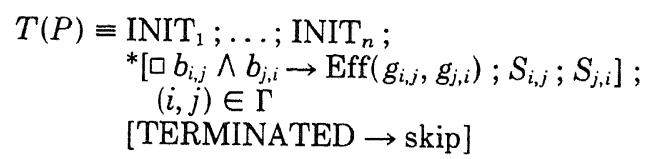

where $\Gamma=\left\{(i, j) ; i \in \Gamma_{j}, j \in \Gamma_{i}, g_{i, j}\right.$, and $g_{j, i}$ match $\}$.

Note that upon exit of the main loop of $T(P)$, BLOCKED holds (which does not necessarily imply TERMINATED). It is easy to see that $P$ and $T(P)$ are ACM Transactions on Programming Languages and Systems, Vol. 8, No. 3, July 1986. 
equivalent in the sense of partial correctness semantics (i.e., when divergence, failures, and deadlocks are not taken into account). Now both rules 1 and 2 exploit these equivalences.

Now consider rule 3. In a deadlock situation every process is either at the main loop entry or has terminated. Thus a global invariant holds in a deadlock situation. Moreover, the formula BLOCKED $\wedge \neg$ TERMINATED holds in a deadlock situation as well. Thus the premises of rule 3 do indeed ensure that no deadlock (relative to $p$ ) can arise.

Finally, rule 4 is sound because auxiliary variables affect neither the control flow of the program (by requirement (i)) or the values of the other variables (by requirement (ii)).

It is worthwhile to point out that the rule of auxiliary variables is not needed in the correctness proofs. This follows from two facts. First, it is not needed in the context of nondeterministic programs, as the theoretical completeness results show (see [1]). And second, due to the equivalence between $P$ and $T(P)$ and the form of the rules, every correctness proof of $T(P)$ can be rewritten as a correctness proof of $P$. However, as we have seen in the previous section, this rule is very helpful in concrete correctness proofs.

It is true that the proposed proof method can only be applied to CSP programs in a normal form. On the other hand, many CSP programs exhibit this form.

Let us now relate our proof method to two other approaches to proving correctness of CSP programs-those of Apt, Francez, and De Roever [3] and of Manna and Pnueli [12].

When discussing the first approach, it is more convenient to consider its simplified and more comprehensive presentation given in [2]. The approach of [2] is based on the use of proofs from a set of assumptions. First, correctness of individual processes is proved from the assumptions about its subparts containing I/O commands (called bracketed sections). When proving correctness of a whole program, these assumptions are discarded when they satisfy a certain test (called a cooperation test).

Now consider a CSP program in the special form with all $\mathrm{INIT}_{i}$ parts being empty. Let each branch of the main loop constitute a bracketed section. Given a bracketed section, $\langle S\rangle$, associated with a branch that starts with a Boolean condition $b$ within the text of process $P_{i}$, choose the assumption $\{b\}\langle S\rangle\{$ true $\}$ for the proof of the $\{$ true $\} P_{i}$ \{TERMINATED $\left.{ }_{i}\right\}$. Then it is easy to see that

$$
A_{i} \vdash\{\text { true }\} P_{i}\{\text { TERMINATED }\} \text {, }
$$

where $A_{i}$ stands for the set of chosen assumptions and ... . . denotes provability in the sense of partial correctness from a set of hypotheses. Now the premises of rule 1 are equivalent to the set of conditions stating that the chosen sets of assumptions cooperate w.r.t. the global invariant $I$. In their presentation, use of the communication axiom, formation rule, and arrow rule is combined.

This shows that (under the assumption that all $\mathrm{INIT}_{i}$ parts are empty) proof rule 1 can be derived in the proof system considered in [2]. This provides another, very indirect proof of its soundness.

Consider now proof rule 2. The main difference between this rule and the corresponding set of rules of [2] is that termination is proved here in a global 
fashion-expression $t$ can contain variables from various processes. To cast this reasoning into the framework of [2], one needs to consider for each process $P_{i}$ a modified version of $t$ in which variables of other processes are replaced by auxiliary variables. Once this is done, the premises of rule 2 can be reformulated appropriately and rule 2 can be derived.

Now proof rule 3 is nothing else but a succinct reformulation of the corresponding approach of [2] where the bracketed sections are chosen as above.

The way the $\mathrm{INIT}_{i}$ parts are handled is based on the observation that these program sections can be moved outside the scope of the parallel composition. In the terminology of Elrad and Francez [6], [INIT ${ }_{1}\|\ldots\|$ INIT $\left._{n}\right]$ is a communication-closed layer of the original program.

In the approach of [2] and [3], bracketed sections can be chosen in a different way, thus shifting slightly the emphasis from global to more local reasoning (for example by reducing $I_{11}$ to a local loop invariant). This cannot be done in the framework of the method proposed here.

Comparison with [12] can be made in a more succinct way. In [12], two types of transitions are considered in the case of CSP programs: local transitions and communication transitions. All proof rules refer to this set of transitions. When applied to CSP programs, their INV-rule becomes very similar to our rule 1 . The main difference is that in our framework the only allowed transitions are those consisting of the joint execution of a pair of branches of the main loops with matching I/O guards. Such a choice of transitions does not make much sense in the framework of [12], where programs are presented in a flowchart-like form and thus have no structure. Appropriate combinations of IND and TRNS rules become from this point of view counterparts of rules 2 and 3 .

The main difference lies in the way properties 3 and 4 stated in Section 2 are proved. According to the terminology of temporal logic (see, e.g., [12]), these are liveness properties. Using temporal logic they are proved by setting up a chain of inevitable events leading to the final one which is to hold eventually. In our approach, liveness properties are formulated as a combination of a weak total correctness statement and a deadlock freedom. This results in a different presentation of the proof.

From this discussion, it becomes clear that the proof method presented in Section 4 does not differ in essence from the approaches of $[2,3]$ and [12]. It simply exploits the particular form of CSP programs to which it is restricted.

\section{ACKNOWLEDGMENTS}

We would like to thank C. Delporte-Gallet and A. Pnueli for their interesting and helpful discussions on the subject of this paper. Also, we are grateful to all three referees for their detailed reports.

\section{REFERENCES}

1. APT, K. R. Ten years of Hoare's logic; a survey, part II. Theor. Comput. Sci. 28 (1984), 83-109.

2. APT, K. R. Proving correctness of CSP programs: a tutorial. In Proceedings of the International Summer School "Control Flow and Data Flow: Concepts of Distributed Programming," NATO ASI Series, vol. F14, M. Broy, Ed., Springer-Verlag, New York, 1985, 441-474.

3. Apt, K. R., Francez, N., AND De Roever, W.P. A proof system for Communicating Sequential Processes. ACM Trans. Program. Lang. Syst. 2, 3 (1980), 359-385. 
4. Apt, K. R., AND Richier, J. L. Real-time clocks versus virtual clocks. In Proceedings of the International Summer School "Control Flow and Data Flow: Concepts of Distributed Programming,” NATO ASI Series, vol. F14, M. Broy, Ed., Springer-Verlag, 1985, 475-503.

5. Dijkstra, E. W., Feijen, W. H., and van Gasteren, A. J. M. Derivation of a termination detection algorithm for distributed computations. Inf. Process. Lett. 16, 5 (1983), 217-219.

6. Elrad, T. E., and Francez, N. Decomposition of distributed programs into communicationclosed layers. Sci. Comput. Program. 2, 3 (1982), 155-174.

7. Francez, N. Distributed termination. ACM Trans. Program. Lang. Syst. 2, 1 (1980), 42-55.

8. Francez, N., Rodeh, M., And Sintzoff, M. Distributed termination with interval assertions. In Proceedings of the International Colloquium on Formalization of Programming Concepts, Lecture Notes in Computer Science, 107, (Peniscola, Spain), Springer-Verlag, New York, 1981.

9. Grumberg, O., Francez, N., Makowsky, J., AND De Roever W. P. A proof rule for fair termination of guarded commands. In Algorithmic Languages, J. W. de Bakker and J. C. Van Vliet, Eds., North-Holland, Amsterdam, 1981, 399-416.

10. Hoare, C. A. R. Communicating sequential processes. Commun. ACM 21, 8 (1978), 666-677.

11. LAMPORT, L. Time, clocks, and the ordering of events in a distributed system, Commun. ACM 21, 7 (1978), 558-565.

12. Manna, Z., ANd PNUeli, A. How to cook a temporal proof system for your pet language. In Proceedings of the Symposium on Principles of Programming Languages (Austin, Tex., 1983), ACM, New York.

13. OWICKI, S., AND GRIES, D. An axiomatic proof technique for parallel programs I. Acta Inf. 6,1 (1976), 319-340.

14. Topor, R. W. Termination detection for distributed computations. Inf. Process. Lett. 18, 1 (1984), 33-36.

Received November 1984; revised December 1985; accepted December 1985. 